\title{
New Type Superconducting Bulk Magnet by Pulse Field Magnetizing With Usable Surface on Both Sides in Open Space
}

\author{
Hiroyuki Fujishiro, Atsushi Fujiwara, Tatsuya Tateiwa, Tetsuo Oka, and Hidemi Hayashi
}

\begin{abstract}
A new-type of magnet system using high- $T_{\mathrm{c}}$ superconducting bulks has been developed, in which two aligned bulk disks are cooled down from the side face by thermal conduction and are magnetized along the $c$-axis in turn employing a split-type pulse coil. The temperature rise $\Delta T$ due to the magnetic flux intrusion is relatively small and the trapped field $B_{\mathrm{T}}^{\mathrm{P}}$ is enhanced in comparison with the conventional bulk magnet system based on a solenoid-type pulse coil. $B_{\mathrm{T}}^{\mathrm{P}}=3.20 \mathrm{~T}$ on the $\mathrm{SmBaCuO}$ bulk surface at $50 \mathrm{~K}$ and $B_{\mathrm{T}}^{4 \mathrm{~mm}}=1.97 \mathrm{~T}$ on the vacuum sheath, $4 \mathrm{~mm}$ above the bulk surface, have been attained. This type of magnet system, consisting of multi-bulks, with usable surfaces on both sides in open space, can be realized only by use of a split-type pulse coil and has a potential for new application fields.
\end{abstract}

Index Terms-Bulk magnet system, high- $T_{c}$ bulk superconductor, pulse field magnetizing, split coil, temperature rise, trapped field.

\section{INTRODUCTION}

$\mathbf{F}$ OR THE PRACTICAL applications of a high- $T_{\mathrm{c}}$ superconducting bulk, a high-strength bulk magnet in a magnetic separation system for waste water etc. is one of the typical models [1]. On magnetizing the bulk, a field cooled magnetizing (FCM) has been usually used. Recently, a pulse field magnetizing (PFM) has been investigated and developed because of a compact and inexpensive experimental setup. The trapped field $B_{\mathrm{T}}^{\mathrm{P}}$ by PFM is, however, generally smaller than that attained by FCM due to the large temperature rise $\Delta T$ during the PFM process. Several approaches have been performed in order to reduce $\Delta T$ and enhance $B_{\mathrm{T}}^{\mathrm{P}}$ [2]-[5]. Very recently, we have attained the trapped field of $B_{\mathrm{T}}^{\mathrm{P}}=4.47 \mathrm{~T}$ by the modified multi-pulse technique combined with stepwise cooling (MMPSC) method, which is the present highest value by PFM [6].

To realize a high-strength bulk magnet, a high- $T_{c}$ bulk is usually cooled down below the superconducting transition temperature $\left(T_{c}\right)$ along the $c$-axis on the cold stage of the helium re-

Manuscript received September 18, 2005. This work was supported in part by Grant-in-Aid for Scientific Research 17560001 from the Ministry of the Education, Culture, Sports, Science and Technology, Japan and from Iwate Prefecture, Japan.

H. Fujishiro, A. Fujiwara, and T. Tateiwa are with the Faculty of Engineering, Iwate University, 4-3-5 Ueda, Morioka 020-8551, Japan (e-mail: fujishiro@iwate-u.ac.jp; t3204017@iwate-u.ac.jp; t.tatei@ezweb.ne.jp).

T. Oka is with IMRA Material R\&D Co., Ltd., 5-50 Hachiken-cho, Kariya 448-0021, Japan (e-mail: toka@ai-i.aisin.co.jp).

H. Hayashi is with Kyushu Electric Power Co., Inc., 2-1-47 Shiobaru, Fukuoka 815-8520, Japan (e-mail: Hidemi_Hayashi/KYUDEN@kyuden.co. jp).

Digital Object Identifier 10.1109/TASC.2006.871278 frigerator and then magnetized also along the $c$-axis using a solenoid-type coil. In this setup, only one bulk can be utilized for one refrigerator, and only the upper surface of the bulk can be utilized as a magnetic pole in open space. Oka et al. constructed a multi-bulk magnet system consisting of 7 bulks and attained $1.4 \mathrm{~T}$ at the center of pole surface in open space [7]. The bulks were mounted on the stainless steel disk $150 \mathrm{~mm}$ in diameter and were cooled down to $67 \mathrm{~K}$ using a Stirling pulse tube cooler. The bulks were simultaneously magnetized by FCM from $5 \mathrm{~T}$ using a cryocooled superconducting magnet with bore $300 \mathrm{~mm}$ in diameter. In a similar setup, it is very difficult to magnetize the multi-bulks by PFM at a time because of the problems of the mechanical strength of the supporting parts and capacity of the power supply. If a magnet system consisting of multi-bulks could be easily realized by successive PFM, a new field of industrial application should develop.

In this paper, we propose and test a new-type of the high- $T_{\mathrm{c}}$ bulk magnet system, in which two bulk disks are mounted and cooled down from the side face along the $a b$-plane, and are magnetized in turn by PFM along the $c$-axis using a split-type pulse coil. We can effectively use both surfaces of each bulk as magnetic poles in open space, which may be a great merit. We compare with the results of a conventional-type bulk magnet system, where the bulk is cooled down along the $c$-axis and magnetized by PFM using a solenoid-type pulse coil.

\section{EXPERIMENTAL PROCEDURE}

Highly $c$-axis oriented $\mathrm{SmBaCuO}$ and $\mathrm{YBaCuO}$ bulk disks (by Dowa Mining Co., Ltd.) $46 \mathrm{~mm}$ in diameter and $15 \mathrm{~mm}$ in thickness were used in this study [5]. The c-axis was perpendicular to the disk surface. The bulk was uniformly impregnated with epoxy resin in vacuum, and the epoxy resin layers on the bulk surface were removed in order to promote the thermal response. Fig. 1 shows the experimental setup around the bulks. Two bulk disks were tightly fastened with the brass-metal bulk holder from the side face and the brass holder was attached to the cold stage of a Gifford McMahon (GM) cycle helium refrigerator (AISIN, GR103). This cooling procedure is expected to reduce the cooling time and the recovering time of temperature after the heat generation by PFM, because the $a b$-plane thermal conductivity $\kappa_{\mathrm{ab}}$ is much higher than the $c$-axis $\kappa_{\mathrm{c}}$ [8]. The adiabatic vacuum cell was of sheath shape with two parallel surfaces situated $4 \mathrm{~mm}$ above each bulk surface. The initial temperature of the cold stage $T_{\mathrm{s}}$ was fixed at $50 \mathrm{~K}$ before every pulse application. The time evolution of temperatures $T 1-T 3$ and the local field $B_{\mathrm{L}}(\mathrm{C})$ on the $\mathrm{SmBaCuO}$ bulk and $T 4$ on 


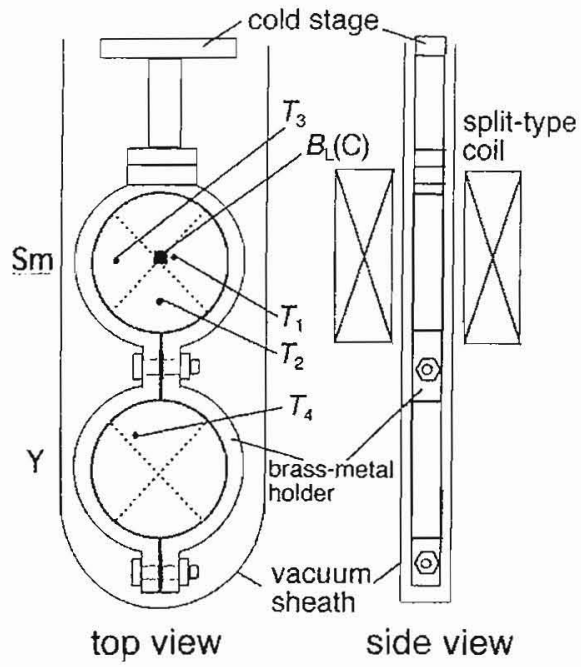

Fig. 1. The experimental setup around the $\mathrm{SmBaCuO}$ and $\mathrm{YBaCuO}$ bulks and the split-type coil ("parallel-sheath-type" system). The positions for the temperature $\left(T 1-T_{4}\right)$ and trapped field $\left(B_{\mathrm{L}}(\mathrm{C})\right)$ measurements on the surface of the bulks are shown.

the $\mathrm{YBaCuO}$ bulk were monitored using the fine chromel-constantan thermocouples and the Hall sensor (F.W.Bell, Model BHA921) adhered in the respective places. The bulks were magnetized using the split-type pulse coil dipped in liquid $N_{2}$. A set of soft iron yokes was located at the center of the split-type coil. The rise time of the pulse field was about $20 \mathrm{~ms}$. The strength of the applied field $B_{\mathrm{ex}}$ was determined from the current flowing through the coil, which ranged from $3.83 \mathrm{~T}$ to 6.41 T. The trapped field distribution $B_{\mathrm{T}}^{4} \mathrm{~mm}, 4 \mathrm{~mm}$ above the bulk surface, was monitored using an axial-type Hall sensor, which was scanned on the vacuum sheath stepwise with a pitch of 1.0 $\mathrm{mm}$. After magnetizing the $\mathrm{SmBaCuO}$ bulk, the split-type coil was moved parallel and the $\mathrm{YBaCuO}$ bulk was magnetized. Hereafter, we abbreviate this type of new magnet system as a "parallel-sheath-type". For comparison, we also magnetized the SmBaCuO bulk at $T_{\mathrm{s}}=50 \mathrm{~K}$ for various $B_{\text {ex }}$ using a solenoid-type pulse coil, as shown in the inset of Fig. 3, and measured $T 1(t), T 2(t)$ and the trapped field $B_{\mathrm{T}}(\mathrm{C})$. We abbreviate the magnet system employing the solenoid coil as a "conventional-type".

\section{RESULTS AND DISCUSSION}

Figs. 2(a) and 2(b) show the time evolutions of temperatures $T 1(t) \sim T 4(t)$ and local field $B_{\mathrm{L}}(\mathrm{C})(t)$ after applying the pulse field of $4.57 \mathrm{~T}$ and $6.09 \mathrm{~T}$ to the $\mathrm{SmBaCuO}$ bulk, respectively. The inset of each figure shows the distribution of $B_{\mathrm{T}}^{4} \mathrm{~mm}$. It can be seen that the maximum temperature rise $\Delta T_{\max }$, the time evolution of temperature, and the $B_{\mathrm{T}}^{4} \mathrm{~mm}$ profile change depending on the $B_{\text {ex }}$ value. In Fig. 2(a) for $B_{\text {ex }}=4.57 \mathrm{~T}, \mathrm{~T} 1(t)$ and $T 3(t)$ rise up sharply with a characteristic peak at $t=2 \mathrm{~s}$ and then rapidly decrease to finally return to $T_{\mathrm{s}} \sim 50 \mathrm{~K}$ in 10 min. On the other hand, $T 2(t)$ increases moderately. The rapid rise and sharp peak in $T 1(t)$ and $T 3(t)$ suggest that the intense heat generation due to the pining loss $Q_{\mathrm{p}}$ and the viscous loss $Q_{v}$ takes place in the vicinity of these positions and that there exists an easy path for the flux motion due to relatively weaker pinning force $F_{\mathrm{p}}$. The $B_{\mathrm{L}}(\mathrm{C})(t)$ quickly saturates within $0.2 \mathrm{~s}$,
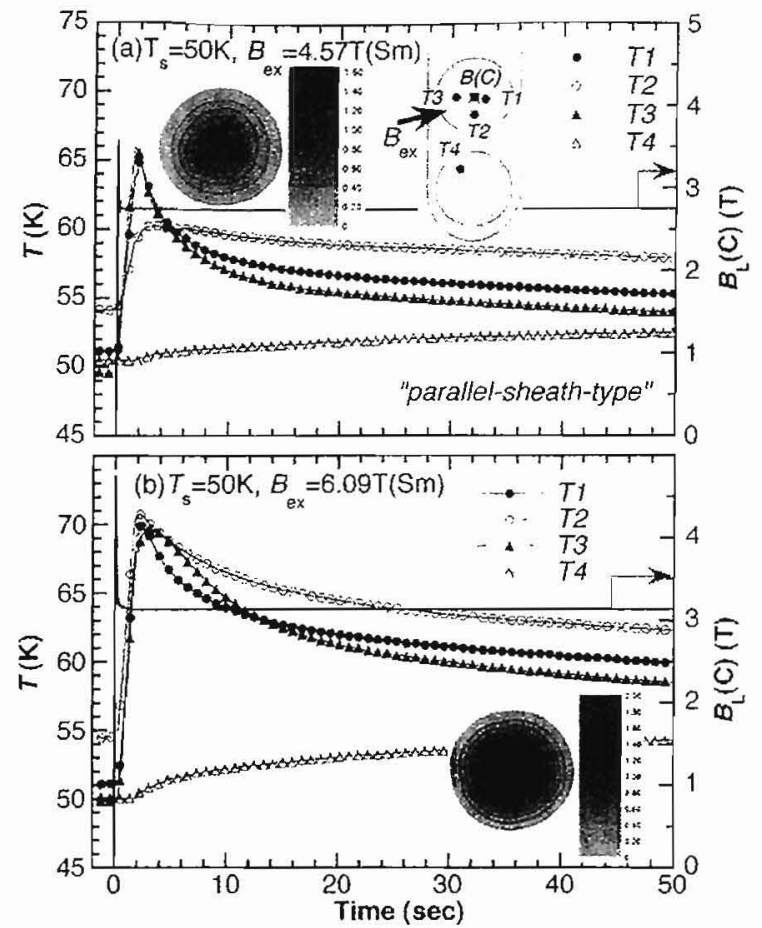

Fig. 2. The time evolution of temperatures $T 1-T 4$ and the local field $B_{\mathrm{L}}(\mathrm{C})$ after applying the pulse field of (a) $4.70 \mathrm{~T}$ and (b) $6.09 \mathrm{~T}$ to the $\mathrm{SmBaCuO}$ bulk using the "parallel-sheath-type" system. The inset shows the trapped field distribution $B_{T}^{4} \mathrm{~mm}$

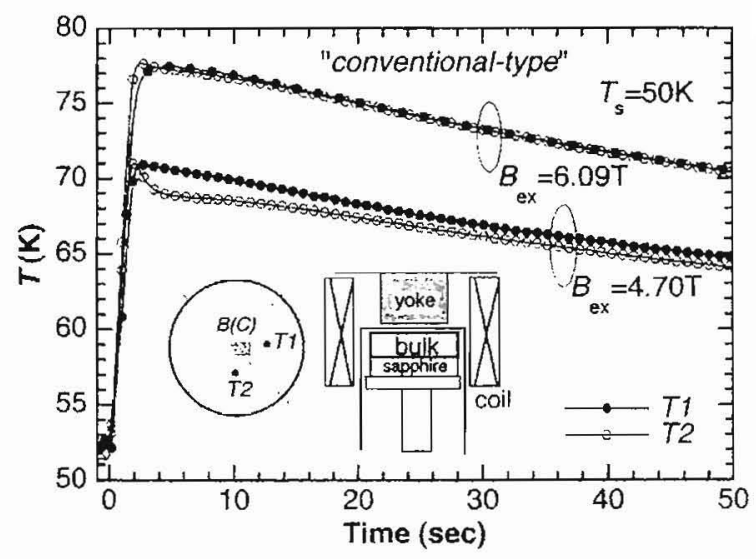

Fig. 3. The time evolutions of temperatures $T 1$ and $T \cdot 2$ of the SmBaC'uO bulk after applying the pulse field of $4.70 \mathrm{~T}$ and $6.09 \mathrm{~T}$ using the "conventional-rype" bulk magnet system. The experimental setup around the bulk and the arrangement of the solenoid-type magnet are shown in the inset.

and the trapped field $B_{\mathrm{T}}^{\mathrm{P}}\left(=B_{\mathrm{L}}(t \rightarrow \infty)\right)$ at the bulk center is $2.75 \mathrm{~T}$. In Fig. $2(\mathrm{~b})$ for $B_{\mathrm{ex}}=6.09 \mathrm{~T}, T 1(t), T 2(t)$ and $T 3(t)$ all rise up faster with a clear peak and the temperature rise increases. $\Delta T_{\max }$ increases with increasing $B_{\mathrm{ex}}$ and the spatial distribution of the heat generation and the $B_{\mathrm{T}}^{4} \mathrm{~mm}$ distribution change depending on $B_{\text {ex }} . B_{\mathrm{T}}^{\mathrm{P}}$ is $3.10 \mathrm{~T}$. $T 4(t)$ on the $\mathrm{YBaCuO}$ bulk increases very slowly. $\Delta T_{\max }$ in $T 4$ is about 1 $\mathrm{K}$ for $B_{\text {ex }}=4.70 \mathrm{~T}$ and slightly increases with increasing $B_{\text {ex }}$. A part of the heat generated in the $\mathrm{SmBaCuO}$ bulk transfers to the neighboring $\mathrm{YBaCuO}$ through the brass-metal holder.

Using the "conventional-type" bulk magnet system, we measured the time evolution of temperatures and trapped field of the present $\mathrm{SmBaCuO}$ bulk at $T_{\mathrm{s}}=50 \mathrm{~K}$ for various $B_{\text {ex }}$. Fig. 3 

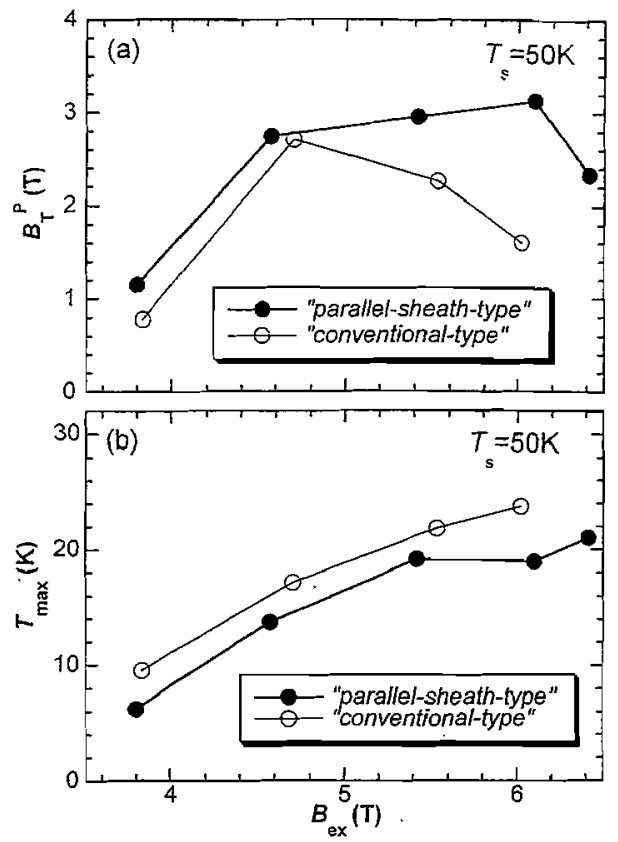

Fig. 4. (a) The trapped field $B_{\mathrm{T}}^{\mathrm{P}}$ and (b) the maximum temperature rise $\Delta T_{\max }$ at the center of the $\mathrm{SmBaCuO}$ bulk as a function of the applied field $B_{\mathrm{ex}}$ using the "parallel-sheath-type" and the "conventional-type" bulk magnet systems.

shows the results for $T 1(t), T 2(t)$ and $B_{\mathrm{L}}(\mathrm{C})(t)$ after applying $B_{\text {ex }}=4.70 \mathrm{~T}$ and $6.09 \mathrm{~T}$. It should be noticed that the time evoIutions of temperature $T 1(t), T 2(t)$ are quite different for the "parallel-sheath-type" system; after rising up to the maximum value, the decay of temperature is more moderate and $\Delta T_{\max }$ is larger. These results may mainly come from the difference in the cooling direction for the bulk; the propagating speed of the generated heat in the $a b$-plane is much higher than that toward the $c$-axis [8]. The generated heat spreads in the high-thermal-diffusive $a b$-plane and more rapidly transfers to the brass holder in the "parallel-sheath-type" system. On the other hand, in the "conventional-type" system, the generated heat flows out along the low-thermal-diffusive ( $c$-axis) direction. We reported the effect of the stainless steel ring setting onto the high- $T_{\mathrm{c}}$ bulk disk on $B_{\mathrm{T}}^{\mathrm{P}}$ and $\Delta T$ during PFM [4]. The ring acts ás a heat reservoir and a part of generated heat in the bulk transfers to the ring. As a result, $\Delta T$ in the bulk decreased and $B_{\mathrm{T}}^{\mathrm{P}}$ was enhanced. In the "parallel-sheath-type" system, as shown in Fig. 1, the brass bulk holder also acts as an effective heat reservoir.

Figs. 4(a) and 4(b) compare the trapped field $B_{\mathrm{T}}^{\mathrm{P}}$ and the maximum temperature rise $\Delta T_{\max }$ at the center of the $\mathrm{SmBaCuO}$ bulk as a function of the applied field $B_{\text {ex }}$ for the "parallelsheath-type" and the "conventional-type" bulk magnet system. In Fig. 4(a), for the "parallel-sheath-type" system, $B_{\mathrm{T}}^{\mathrm{P}}$ increases with increasing $B_{\mathrm{ex}}$ up to $6.09 \mathrm{~T}$ and then decreases for $B_{\mathrm{ex}}=$ 6.41 T. On the other hand, in the "conventional-type" systems, $B_{\mathrm{T}}^{\text {P }}$ takes a maximum at $B_{\text {ex }}=4.70 \mathrm{~T}$ and then decreases with the further increase of $B_{\mathrm{ex}}$. $B_{\mathrm{T}}^{\mathrm{P}}$ for the "parallel-sheath-type" system is always larger than that for the "conventional-type" one and does not show a saturation tendency up to $B_{\mathrm{ex}}=6.09 \mathrm{~T}$. In Fig. 4(b), $\Delta T_{\max }$ increases with increasing $B_{\text {ex }}$ for both systems. However, the $\Delta T_{\max }$ value for the "parallel-sheath-type" system is always smaller than that for the "conventional-type"

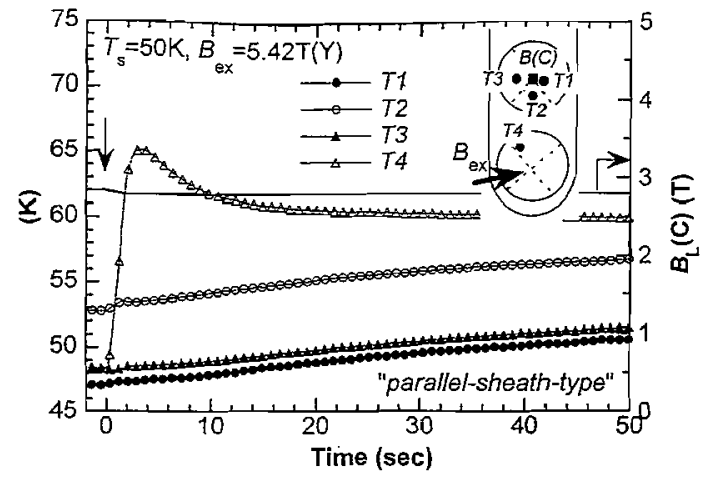

Fig. 5. $T 4(t)$ on the $\mathrm{YBaCuO}$ bulk and $T 1(t)-T 3(t)$ and $B_{\mathrm{L}}(\mathrm{C})(t)$ on $\mathrm{SmBaCuO}$ bulk after applying $B_{\mathrm{ex}}=5.42 \mathrm{~T}$ to the $\mathrm{YBaCuO}$ bulk. The $\mathrm{SmBaCuO}$ bulk has already been magnetized by $B_{\text {ex }}=6.09 \mathrm{~T}$ and the $B_{\mathrm{T}}^{\mathrm{P}}=$ $2.90 \mathrm{~T}$ has been trapped at the bulk center.

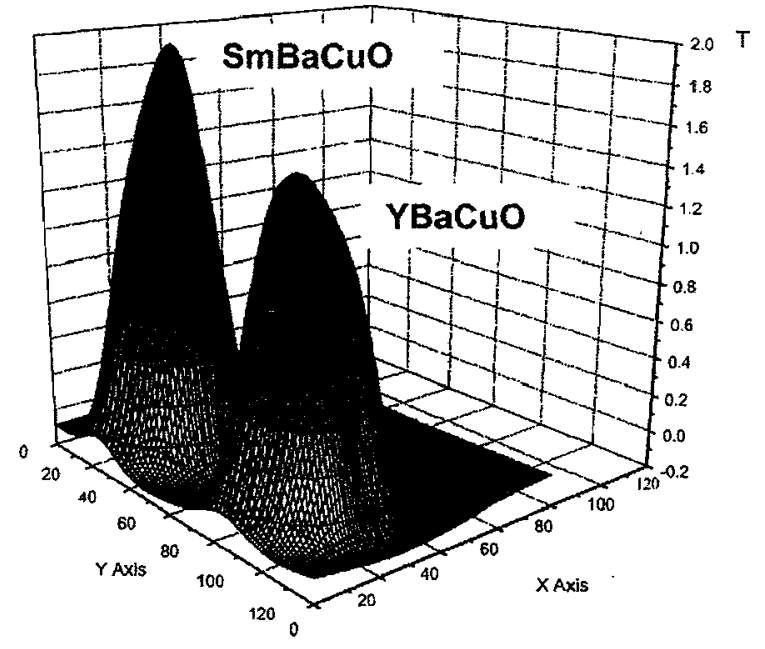

Fig. 6. The trapped field distribution $B_{T}^{4 m m}$ obtained by the "parallel-sheathtype" bulk magnet system. After applying the $B_{\text {ex }}=6.09 \mathrm{~T}$ to the $\mathrm{SmBaCuO}$ bulk, the split-type coil was moved parallel and the puise field of $B_{\mathrm{ex}}=5.42 \mathrm{~T}$ was applied to the $\mathrm{YBaCuO}$ bulk at $T_{\mathrm{s}}=50 \mathrm{~K}$.

system. These results suggest that the effect of the heat reservoir of the brass holder is significant and, resultantly, $\Delta T_{\max }$ decreases and $B_{T}^{\mathrm{P}}$ increases, in comparison with the "conventional-type" system.

By use of the "parallel-sheath-type" system, we performed the PFM procedures for two bulks sequentially; at first, the $\mathrm{SmBaCuO}$ bulk. was magnetized by $B_{\mathrm{ex}}=6.09 \mathrm{~T}$ at 50 $\mathrm{K}$ and then the split-type coil was moved parallel toward the $\mathrm{YBaCuO}$ bulk and the magnetic field of $B_{\mathrm{ex}}=5.42 \mathrm{~T}$. was applied to the $\mathrm{YBaCuO}$ bulk. Fig. 5 presents $T 4(t)$ on the $\mathrm{YBaCuO}$ bulk and $T 1(t)-T 3(t)$ and $B_{\mathrm{L}}(C)(t)$ on the $\mathrm{SmBaCuO}$ bulk after applying the pulse field. $T 4(t)$ sharply increases $\left(\Delta T_{\max }=17 \mathrm{~K}\right)$, takes a maximum at $t=3 \mathrm{~s}$ and then decreases. $T 1(t)-T 3(t)$ gradually increase by $2 \sim 3 \mathrm{~K}$. It should be noted that the $B_{\mathrm{L}}(\mathrm{C})(t)$ of the $\mathrm{SmBaCuO}$ bulk slightly decreases after applying the pulse field to the $\mathrm{YBaCuO}$ bulk due to the small temperature rise in the $\mathrm{SmBaCuO}$ bulk. These results suggest that there is almost no deterioration of $B_{\mathrm{T}}^{\mathrm{P}}$ trapped on the neighboring bulk in the present case with the $61 \mathrm{~mm}$ distance between the centers of two bulks. Fig. 6 shows the trapped field distribution of $B_{\mathrm{T}}^{4} \mathrm{~mm}$ in this case. We 


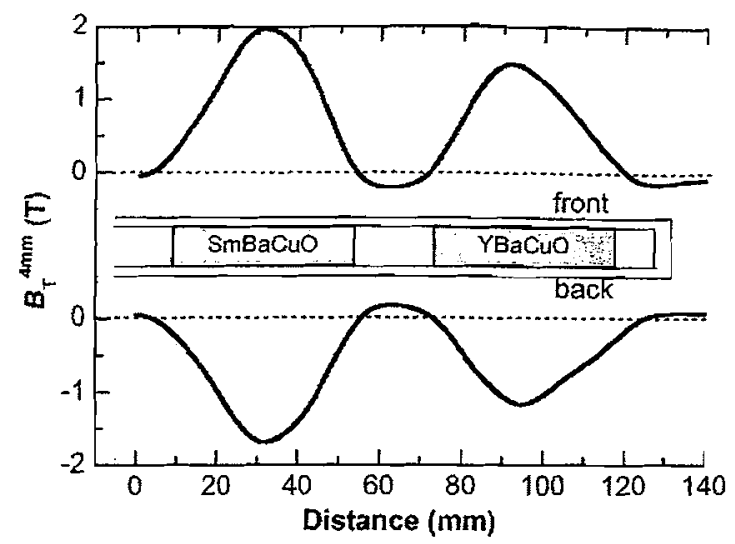

Fig. 7. The line scan profiles of the $B_{\mathrm{T}}^{4 \mathrm{~mm}}$ value along the direction through the bulk center on both "front" and "back" surfaces.

can confirm the conical $B_{\mathrm{T}}^{4 \mathrm{~mm}}$ profiles for both bulks. The maximum $B_{\mathrm{T}}^{4}{ }^{\mathrm{mm}}$ value is $1.97 \mathrm{~T}$ and $1.49 \mathrm{~T}$ for the $\mathrm{SmBaCuO}$ and $\mathrm{YBaCuO}$ bulk, respectively. The smaller $B_{\mathrm{T}}^{4} \mathrm{~mm}$ value in the $\mathrm{YBaCuO}$ bulk comes from the lower $J_{c}$ value of this bulk [5].

Fig. 7 shows the line scan profiles of the $B_{T}^{4} \mathrm{~mm}$ value along the direction through the bulk centers on both surfaces of the vacuum sheath. In this figure, "front" means the sample surface side on which the seed crystal was attached during the crystal growth and "back" means the opposite side. The very similar $B_{\mathrm{T}}^{4 \mathrm{~mm}}$ profiles can be seen on both surfaces in open space. However, the $B_{\mathrm{T}}^{4} \mathrm{~mm}$ value on the "front" surface is a little bit larger than that on the "back" surface due to the difference of crystalline property of the superconducting bulk.

\section{CONCLUSION}

A new-type of magnet system consisting of two high- $T_{\mathrm{c}}$ bulks, named as "parallel-sheath-type", with usable surface on both sides in open space, has been realized employing a split-type pulse magnet. In the "parallel-sheath-type" magnet system, the temperature rise $\Delta T$ due to the magnetic flux intrusion is relatively small and the trapped field $B_{\mathrm{T}}^{\mathrm{P}}$ is enhanced compared with the "conventional-type" bulk magnet system based on a solenoid-type pulse coil. $B_{\mathrm{T}}^{\mathrm{P}}=3.20 \mathrm{~T}$ on the $\mathrm{SmBaCuO}$ bulk surface at $50 \mathrm{~K}$ and $B_{\mathrm{T}}^{4} \mathrm{~mm}=1.97 \mathrm{~T}$ on the vacuum sheath in open space have been attained. This type of magnet system consisting of multi-bulks can be realized only by the PFM technique using a split-type pulse coil and has a potential for new application fields. The extension of the "parallel-sheath-type" system to more than 3 bulks is possible without serious difficulty.

\section{ACKNOWLEDGMENT}

The authors greatly thank Prof. M. Ikebe for the valuable discussion and M. Kaneyama and K. Kawai of Iwate University for the technical support.

\section{REFERENCES}

[1] H. Hayashi, K. Tsutsumi, N. Saho, N. Nishizima, and K. Asano, "Study on a mobile-type magnetic separator applying high- $T_{\mathrm{c}}$ bulk superconductors," Phys. C, vol. 392-396, pp. 745-748, 2003

[2] U. Mizutani, T. Oka, Y. Itoh, Y. Yanagi, M. Yoshikawa, and H. Ikuta "Pulse-field magnetization applied to high- $T_{\mathrm{c}}$ superconductors," Appl. Supercond., vol. 6, pp. 235-246, 1998.

[3] M. Sander, U. Sutter, R. Koch, and M. Kläser, "Pulsed magnetization of HTS bulk at $T<7 T \mathrm{~K}$," Supercond. Sci. Technol., vol. 13, pp $841-845,2000$.

[4] H. Fujishiro, K. Yokoyama, M. Kaneyama, T. Oka, and K. Noto, "Effect of metal ring setting outside HTSC bulk disk on trapped field and temperature rise in pulse field magnetizing," IEEE Trans. Appl. Supercond., vol. 15, pp. 3762-3765, 2005.

[5] H. Fujishiro, M. Kaneyama, K. Yokoyama, T. Oka, and K. Noto, "Risetime elongation effects on trapped field and temperature rise in pulse field magnetization for high temperature superconducting bulk,"Jpn. J. Appl. Phys., vol. 44, pp. 4919-4925, 2005.

[6] H. Fujishiro, M. Kaneyama, T. Tateiwa, and T. Oka, "A record high trapped magnetic field by pulse field magnetization using $\mathrm{GdBaCuO}$ bulk superconductor," Jpn. J. Appl. Phys., vol. 44, pp. L1221-L1224, 2005.

[7] T. Oka, K. Yokoyama, and K. Noto, "Construction of strong magnetic field generators by high $T_{c}$ bulk superconductors and its applications," IEEE Trans. Appl. Supercond., vol. 14, pp. 1058-1061, 2004.

[8] H. Fujishiro and S. Kohayashi, "Thermal conductivity, thermal diffusivity and thermoelectric power in Sm-based bulk superconductors," IEEE Trans. Appl. Supercond., vol. 12, pp. 1124-1127, 2002. 